\title{
On the generalized Hartley-Hilbert and Fourier-Hilbert transforms
}

SKQ Al-Omari ${ }^{1}$ and A Kılıçman ${ }^{2 *}$

${ }^{\text {"Correspondence: }}$

akilicman@putra.upm.edu.my

${ }^{2}$ Current address: Department of Mathematics and Institute of

Mathematical Research, Universit Putra Malaysia (UPM), 43400 UPM,

Serdang, Selangor, Malaysia

Full list of author information is

available at the end of the article

\begin{abstract}
In this paper, we discuss Hartley-Hilbert and Fourier-Hilbert transforms on a certain class of generalized functions. The extended transforms considered in this article are shown to be well-defined, one-to-one, linear and continuous mappings with respect to $\delta$ and $\Delta$ convergence. Certain theorems are also established.
\end{abstract}

MSC: Primary 54C40; 14E20; secondary 46E25; $20 \mathrm{C} 20$

Keywords: Hilbert transform; Hartley transform; Fourier transform; Hartley-Hilbert transform; Boehmian space

\section{Introduction}

The Hartley transform of a function $f(t)$ of one variable is defined in [1]

$$
\mathfrak{H}(\xi):=(\mathfrak{H} f)(\xi)=\int_{-\infty}^{\infty} f(t) \operatorname{cas}(\xi t) d t
$$

where $\operatorname{cas}(\xi t)=\cos (\xi t)+\sin (\xi t)$ is the transform kernel. The Hartley transform is a spectral transform closely related to the Fourier transform. It contains the same information as the Fourier transform does, and no advantage accrues in its use for complex signals. For real signals, the Hartley transform is real and this can offer computational advantages in the applications of signal processing that traditionally make use of Fourier transforms.

The Hilbert transform of a function $f(t)$ via the Hartley transform (Hartley-Hilbert transform) is described in [2] as

$$
\mathfrak{h h}(t):=(\mathfrak{h h} f)(t):=\frac{1}{\pi} \int_{0}^{\infty}\left(\mathfrak{H}^{o}(\xi) \cos (\xi t)+\mathfrak{H}^{e}(\xi) \sin (\xi t)\right) d \xi,
$$

where

$$
\mathfrak{H}(\xi):=(\mathfrak{H} f)(\xi)=\int_{-\infty}^{\infty} f(t) \operatorname{cas}(\xi t) d t:=\mathfrak{H}^{e}(\xi)+\mathfrak{H}^{o}(\xi),
$$

$\mathfrak{H}^{e}(\xi)$ and $\mathfrak{H}^{o}(\xi)$ being the even and odd components of the Hartley transform [3].

If a casual function $f(t)(f(t)=0, t<0)$ is considered, then even and odd components $\mathfrak{H}^{e}(\xi)$ (respectively, $\mathfrak{H}^{o}(\xi)$ ) of the Hartley transform of $f(t)$ constitute a Hilbert transform pair:

$$
\mathfrak{H}^{o}(\xi)=\frac{1}{\pi} \int_{-\infty}^{\infty} \frac{\mathfrak{H}^{e}(u)}{\xi-u} d u
$$


and

$$
\mathfrak{H}^{e}(\xi)=\frac{1}{\pi} \int_{-\infty}^{\infty} \frac{\mathfrak{H}^{o}(u)}{\xi-u} d u ;
$$

see $[2,4]$.

On the other hand, the Hilbert transform of $f(t)$ via the Fourier transform (FourierHilbert transform) is defined by

$$
\mathfrak{f h}(t):=(\mathfrak{f h} f)(t)=\frac{1}{\pi} \int_{0}^{\infty}(\mathcal{F} \mathcal{I}(\xi) \cos (\xi t)-\mathcal{F} \mathcal{R}(\xi) \sin (\xi t)) d \xi,
$$

where

$$
\mathcal{F}(\xi)=\int_{-\infty}^{\infty} f(t) e^{-i \xi t} d t:=\mathcal{F} \mathcal{R}(\xi)-i \mathcal{F} \mathcal{I}(\xi)
$$

$\mathcal{F} \mathcal{R}(\xi)$ and $\mathcal{F} \mathcal{I}(\xi)$ being the real and imaginary components of the Fourier transform of $f(t)$.

In [5], Fourier and Hartley transforms are coined as mathematical twins. This suggests that the corresponding properties of Fourier-Hilbert and Hartley-Hilbert transforms can simultaneously be developed and the inherited applications may nicely be replaced. It is interesting to know that Hartley-Hilbert and Fourier-Hilbert transforms enjoy wide applications in signal processing, network theory and some other geophysical applications. In [6], it has been shown that Fourier-Hilbert and Hartley-Hilbert transforms, while possessing the same magnitude, differ in phase by $270^{\circ}$. On the other hand, the inverse Hartley-Hilbert transform returns the original function unlike the Fourier-Hilbert transform, which results in the negative of the original function.

In the present article, we further discuss this pair of transforms on certain spaces of generalized functions. We spread results over three sections. Fourier-Hilbert and HartleyHilbert transforms are reviewed in Section 1. The distributional definition of the cited transforms is presented in Section 2. The Hartley-Hilbert transform is extended to the context of Boehmians in Section 3, where some properties are also described.

\section{The extended Hartley-Hilbert and Fourier-Hilbert transforms}

Let $\mathcal{E}(\mathcal{R})$, in short $\mathcal{E}$, be the space of all infinitely smooth complex-valued functions $\varphi(t)$ over $\mathcal{R}$. Each $\varphi(t) \in \mathcal{E}$ should satisfy the set of inequalities

$$
\gamma_{k}(\phi)=\sup _{t \in \mathcal{K}}\left|\mathcal{D}_{t}^{k} \phi(t)\right|<\infty
$$

where $k \in \mathcal{N}(k=0,1,2, \ldots)$ and $\mathcal{K}$ run through compact subsets of $\mathcal{R}, \mathcal{D}_{t} \equiv \frac{d}{d t}$. The topology of $\mathcal{E}(\mathcal{R})$ is generated by the sequence $\left(\gamma_{k}(\phi)\right)_{0}^{\infty}$ of multinorms which makes $\mathcal{E}(\mathcal{R})$ a locally convex Hausdörff topological vector space. Also,

$$
\mathcal{D}(\mathcal{R}) \subset \mathcal{E}(\mathcal{R})
$$

$\mathcal{D}(\mathcal{R})$ is the Schwartz space of test functions. Denoting by $\mathcal{E}^{\prime}(\mathcal{R})$, or $\mathcal{E}^{\prime}$, the space of continuous linear forms on $\mathcal{E}(\mathcal{R})$, the topology of $\mathcal{D}(\mathcal{R})$ is stronger than that induced on $\mathcal{D}(\mathcal{R})$ 
by $\mathcal{E}^{\prime}(\mathcal{R})$, and the restriction of any element of $\mathcal{E}(\mathcal{R})$ to $\mathcal{D}(\mathcal{R})$ is in $\mathcal{D}^{\prime}(\mathcal{R})$, the space of Schwartz distributions; see [7] and [8]. Elements of $\mathcal{E}^{\prime}(\mathcal{R})$ are the so-called distributions of compact supports; see, for example, [4, 9-11] and [6].

Theorem 1 Let $\left(\mathfrak{H} \varphi_{j}\right) \rightarrow 0$ as $j \rightarrow \infty$ in $\mathcal{E}(\mathcal{R})$, then $\mathfrak{H}^{e} \varphi_{j} \rightarrow 0$ and $\mathfrak{H}^{o} \varphi_{j} \rightarrow 0$ in $\mathcal{E}(\mathcal{R})$ as $j \rightarrow \infty$.

Proof The proof of this remark is straightforward from the fact [9, (78), (79)]

$$
\left(\mathfrak{H}^{e} \varphi_{j}\right)(t)=\frac{1}{2}\left(\mathfrak{H} \varphi_{j}(t)+\mathfrak{H} \varphi_{j}(-t)\right)
$$

and

$$
\left(\mathfrak{H}^{o} \varphi_{j}\right)(t)=\frac{1}{2}\left(\mathfrak{H} \varphi_{j}(t)-\mathfrak{H} \varphi_{j}(-t)\right),
$$

thus detailed proof is avoided.

We note that the space of distributions of compact support is closed under differentiation and multiplication by smooth functions, thus we have the following theorem.

Theorem 2 Let $\varphi \in \mathcal{E}(\mathcal{R})$, then $\mathfrak{H}^{e}(\xi) \in \mathcal{E}(\mathcal{R})$.

Proof For every $\varphi \in \mathcal{E}(\mathcal{R})$, we have

$$
\left|\mathcal{D}_{\xi}^{k} \mathfrak{H}^{e}(\xi)\right|=\left|\int_{\mathcal{K}} \varphi(t) \mathcal{D}_{\xi}^{k} \cos (\xi t) d t\right| \leq \int_{\mathcal{K}}\left|t^{k} \varphi(t)\right| d t .
$$

Then by considering supremum over compact subsets $\mathcal{K}$ of $\mathcal{R}$, we get

$$
\sup _{\xi \in \mathcal{K}}\left|\mathcal{D}_{\xi}^{k} \mathfrak{H}^{e}(\xi)\right|<\infty
$$

for all nonnegative integers $k$. This completes the proof of the theorem.

Theorem 3 Let $\varphi \in \mathcal{E}(\mathcal{R})$, then $\mathfrak{H}^{o}(\xi) \in \mathcal{E}(\mathcal{R})$.

Proof Proof is similar to that of Theorem 2 and therefore we prefer to omit details.

Theorem 4 Let $\varphi \in \mathcal{E}(\mathcal{R})$, then $\mathfrak{h h} \varphi \in \mathcal{E}(\mathcal{R})$.

Proof Let $\varphi \in \mathcal{E}(\mathcal{R})$ be arbitrary, then simple computation yields

$$
\left|\mathcal{D}_{t}^{k} \mathfrak{h} h \varphi(t)\right| \leq \frac{1}{\pi} \int_{0}^{\infty}\left|\xi^{k}\right|\left|\mathfrak{H}^{o}(\xi)+\mathfrak{H}^{e}(\xi)\right| d \xi,
$$

where $\mathfrak{H}^{o}(\xi)$ and $\mathfrak{H}^{e}(\xi)$ are the odd and even components of $\mathfrak{H} \varphi$. Theorem 2 and Theorem 3 imply $\mathfrak{H}^{o}(\xi)$ and $\mathfrak{H}^{e}(\xi)$ are in $\mathcal{E}(\mathcal{R})$. The linearity of $\mathcal{E}(\mathcal{R})$ implies $\mathfrak{H}^{o}(\xi)+\mathfrak{H}^{e}(\xi) \in \mathcal{E}(\mathcal{R})$. Hence,

$$
\left|\xi^{k}\right|\left|\mathfrak{H}^{o}(\xi)+\mathfrak{H}^{e}(\xi)\right| \in \mathcal{E}(\mathcal{R}) .
$$


This implies

$$
\int_{0}^{\infty}\left|\xi^{k}\right|\left|\mathfrak{H}^{o}(\xi)+\mathfrak{H}^{e}(\xi)\right| d \xi \in \mathcal{E}(\mathcal{R})
$$

Hence, from (9), we get

$$
\sup _{t \in \mathcal{K}}\left|\mathcal{D}_{t}^{k} \mathfrak{h} \mathfrak{h} \varphi(t)\right|<\infty
$$

for every $k \in \mathcal{N}$, where $\mathcal{K}$ varies over compact subsets of $\mathcal{R}$. Therefore, the theorem is proved.

Now, by considering proofs which are alike to those used for Theorem 1, Theorem 2 and Theorem 3 and further employing the fact that [2]

$$
\mathcal{F} \mathcal{R}(\xi)=\mathfrak{H}^{e}(\xi) \text { and } \mathcal{F} \mathcal{I}(\xi)=\mathfrak{H}^{o}(\xi)
$$

we state the following theorem.

Theorem 5 Let $\varphi \in \mathcal{E}(\mathcal{R})$, then

(i) $\mathcal{F} \mathcal{R}(\xi) \in \mathcal{E}(\mathcal{R})$,

(ii) $\mathcal{F} \mathcal{I}(\xi) \in \mathcal{E}(\mathcal{R})$,

(iii) $\mathfrak{f h} \varphi \in \mathcal{E}(\mathcal{R})$.

As a consequence of Theorem 4 and Theorem 5, we have

Definition 6 Let $f \in \mathcal{E}^{\prime}(\mathcal{R})$, then the extended Hartley-Hilbert and Fourier-Hilbert transforms of a distribution $f$ of compact support are respectively defined as

$$
\langle\mathfrak{h} \mathfrak{h} f, \varphi\rangle=\langle f, \mathfrak{h} \mathfrak{h} \varphi\rangle
$$

and

$$
\langle\mathfrak{f h} f, \varphi\rangle=\langle f, \mathfrak{f h} \varphi\rangle
$$

for every $\varphi \in \mathcal{E}(\mathcal{R})$.

From (10) and (11), we establish that $\mathfrak{h} h f, \mathfrak{f h} f \in \mathcal{E}^{\prime}(\mathcal{R})$ for each $f \in \mathcal{E}^{\prime}(\mathcal{R})$.

Theorem 7 Let $f \in \mathcal{E}^{\prime}(\mathcal{R})$, then the mappings

$$
\langle\mathfrak{h} \mathfrak{h} f, \varphi\rangle=\langle f, \mathfrak{h} \mathfrak{h} \varphi\rangle, \quad \forall \varphi \in \mathcal{E}(\mathcal{R})
$$

and

$$
\langle\mathfrak{f h} f, \varphi\rangle=\langle f, \mathfrak{f h} \varphi\rangle, \quad \forall \varphi \in \mathcal{E}(\mathcal{R})
$$

are continuous mappings from $\mathcal{E}^{\prime}(\mathcal{R})$ onto $\mathcal{E}^{\prime}(\mathcal{R})$. 
Proof We prove (12) since the proof of (13) is quite similar. Let $\varphi \in \mathcal{E}(\mathcal{R})$, then Theorem 3 implies that

$\mathfrak{h h} \varphi \in \mathcal{E}(\mathcal{R})$

Hence, $\langle f, \mathfrak{h h} \varphi\rangle$ is well defined.

Let $f_{1}, f_{2} \in \mathcal{E}^{\prime}(\mathcal{R})$, then $f_{1}+f_{2} \in \mathcal{E}^{\prime}(\mathcal{R})$ and

$$
\left\langle\mathfrak{h} \mathfrak{h}\left(f_{1}+f_{2}\right), \varphi\right\rangle=\left\langle f_{1}+f_{2}, \mathfrak{h} \mathfrak{h} \varphi\right\rangle,
$$

i.e.,

$$
\left\langle\mathfrak{h} \mathfrak{h}\left(f_{1}+f_{2}\right), \varphi\right\rangle=\left\langle f_{1}, \mathfrak{h} \mathfrak{h} \varphi\right\rangle+\left\langle f_{2}, \mathfrak{h} \mathfrak{h} \varphi\right\rangle .
$$

Therefore, $\langle\mathfrak{h} \mathfrak{h} f, \varphi\rangle=\langle f, \mathfrak{h} h \varphi\rangle$ defines a linear functional on $\mathcal{E}(\mathcal{R})$. If $\varphi_{j} \rightarrow 0$ in $\mathcal{E}(\mathcal{R})$ as $j \rightarrow \infty$, then from Theorem $3, \mathfrak{h h} \varphi_{j} \rightarrow 0$ in $\mathcal{E}(\mathcal{R})$ as $j \rightarrow \infty$.

Thus, $\left\langle f, \mathfrak{h} h \varphi_{j}\right\rangle \rightarrow 0$ as $j \rightarrow \infty$, and hence

$$
\left\langle\mathfrak{h h} f, \varphi_{j}\right\rangle \rightarrow 0 \quad \text { as } j \rightarrow \infty .
$$

Therefore, (12) defines a continuous linear functional on $\mathcal{E}(\mathcal{R})$ and thus distribution in $\mathcal{E}^{\prime}(\mathcal{R})$

Moreover, $f_{j} \rightarrow f$ in $\mathcal{E}^{\prime}(\mathcal{R})$ as $j \rightarrow \infty$ implies $\left\langle f_{j}, \varphi\right\rangle \rightarrow\langle f, \varphi\rangle$ as $j \rightarrow \infty$, where $\varphi \in \mathcal{E}(\mathcal{R})$ is arbitrary. Thus,

$$
\begin{aligned}
\left\langle\mathfrak{h h} f_{j}, \varphi\right\rangle & =\left\langle f_{j}, \mathfrak{h h} \varphi\right\rangle \rightarrow\langle f, \mathfrak{h h} \varphi\rangle \\
& =\langle\mathfrak{h} \mathfrak{h} f, \varphi\rangle,
\end{aligned}
$$

i.e.,

$$
\mathfrak{h h} f_{j} \rightarrow \mathfrak{h h} f
$$

as $j \rightarrow \infty$ in $\mathcal{E}^{\prime}(\mathcal{R})$.

This proves (12). Similarly, (13) can be proved. The proof of this theorem is completed.

Denote by $\mathcal{L}^{1}$ the Lebesgue space of integrable functions. Between two $\mathcal{L}^{1}$ functions, $\varphi_{1}$ and $\varphi_{2}$, the convolution product is defined by

$$
\left(\varphi_{1} * \varphi_{2}\right)(\xi)=\int_{-\infty}^{\infty} \varphi_{1}(t) \varphi_{2}(\xi-t) d t
$$

The Fourier transform of the convolution product $\varphi_{1} * \varphi_{2}$ is defined by [4]

$$
\mathcal{F}\left(\varphi_{1} * \varphi_{2}\right)(\xi)=\mathcal{F} \varphi_{1}(\xi) \mathcal{F} \varphi_{2}(\xi) .
$$


The most complicated convolution theorem of the Hartley transform, compared to the Fourier transform, is that [1]

$$
\mathfrak{H}\left(\varphi_{1} * \varphi_{2}\right)(\xi)=\frac{1}{2} \mathfrak{G}\left(\mathfrak{H} \varphi_{1} \times \mathfrak{H} \varphi_{2}\right),
$$

where

$$
\begin{aligned}
\mathfrak{G}\left(\varphi_{1} \times \varphi_{2}\right)(\xi)= & \varphi_{1}(\xi) \varphi_{2}(\xi)+\varphi_{1}(\xi) \varphi_{2}(-\xi) \\
& +\varphi_{1}(\xi) \varphi_{2}(\xi)-\varphi_{1}(-\xi) \varphi_{2}(-\xi) .
\end{aligned}
$$

Following the manipulation theorem of Hartley transform for the Hartley-Hilbert transform is essential for the following investigation.

Theorem 8 Let $\varphi_{1}$ and $\varphi_{2}$ be $\mathcal{L}^{1}$ functions, then

$$
\begin{aligned}
\left(\mathfrak{h h}\left(\varphi_{1} * \varphi_{2}\right)\right)(t)= & \int_{0}^{\infty}\left(\mathfrak{H}_{1}^{e}(\xi) \mathfrak{H}_{2}^{o}(\xi)+\mathfrak{H}_{1}^{o}(\xi) \mathfrak{H}_{2}^{e}(\xi)\right) \cos (\xi t) d \xi \\
& +\int_{0}^{\infty}\left(\mathfrak{H}_{1}^{e}(\xi) \mathfrak{H}_{2}^{e}(\xi)-\mathfrak{H}_{1}^{o}(\xi) \mathfrak{H}_{2}^{o}(\xi)\right) \sin (\xi t) d \xi
\end{aligned}
$$

where the pair $\left(\mathfrak{H}_{1}^{e}, \mathfrak{H}_{1}^{o}\right)\left(\left(\mathfrak{H}_{2}^{e}, \mathfrak{H}_{2}^{o}\right)\right)$ is the even and odd components of Hartley transforms of $\varphi_{1}\left(\varphi_{2}\right)$, respectively.

Proof For every $\varphi_{1}$ and $\varphi_{2}, \mathfrak{h} \mathfrak{h}\left(\varphi_{1} * \varphi_{2}\right)$ is given by

$$
\begin{aligned}
\left(\mathfrak{h h}\left(\varphi_{1} * \varphi_{2}\right)\right)(t) & =\int_{0}^{\infty}\left(\mathfrak{H}^{o}\left(\varphi_{1} * \varphi_{2}\right)(\xi) \cos (\xi t)+\mathfrak{H}^{e}\left(\varphi_{1} * \varphi_{2}\right)(\xi) \sin (\xi t)\right) d \xi \\
& =I_{1}+I_{2}
\end{aligned}
$$

where

$$
I_{1}(t)=\int_{0}^{\infty} \mathfrak{H}^{o}\left(\varphi_{1} * \varphi_{2}\right)(\xi) \cos (\xi t) d \xi
$$

and

$$
I_{2}(t)=\int_{0}^{\infty} \mathfrak{H}^{e}\left(\varphi_{1} * \varphi_{2}\right)(\xi) \sin (\xi t) d \xi .
$$

It suffices to prove that

$$
I_{1}(t)=\int_{0}^{\infty}\left(\mathfrak{H}_{1}^{e}(\xi) \mathfrak{H}_{2}^{o}(\xi)+\mathfrak{H}_{1}^{o}(\xi) \mathfrak{H}_{2}^{e}(\xi)\right) \cos (\xi t) d \xi
$$

and

$$
I_{2}(t)=\int_{0}^{\infty}\left(\mathfrak{H}_{1}^{e}(\xi) \mathfrak{H}_{2}^{e}(\xi)-\mathfrak{H}_{1}^{o}(\xi) \mathfrak{H}_{2}^{o}(\xi)\right) \sin (\xi t) d \xi
$$


To establish (20), we invoke (14) in (18) to get

$$
I_{1}(t)=\int_{0}^{\infty} \int_{-\infty}^{\infty}\left(\int_{-\infty}^{\infty} \varphi_{1}(y) \varphi_{2}(x-y) d y\right) \sin (\xi x) \cos (\xi t) d x d \xi
$$

Fubini's theorem and the substitution $x-y=z$ imply that

$$
I_{1}(t)=\int_{0}^{\infty} \cos (\xi t) \int_{-\infty}^{\infty} \varphi_{1}(y) \int_{-\infty}^{\infty} \varphi_{2}(z) \sin (\xi z+\xi y) d z d y d \xi .
$$

Applying the identity

$$
\sin (\xi z+\xi y)=\sin (\xi z) \cos (\xi y)+\sin (\xi y) \cos (\xi z)
$$

to (23) and using simple computation jointly with Fubini's theorem, we establish (20).

Similarly, by using the Fubini's theorem and the identity

$$
\cos (\xi z+\xi y)=\cos (\xi z) \cos (\xi y)-\sin (\xi z) \sin (\xi y)
$$

together with technique which is similar to that of (20), we derive (21). Hence, the theorem is completely proved.

\section{The Hartley-Hilbert transform of Boehmians}

In this section, we investigate Hartley-Hilbert transform since proofs involving FourierHilbert transform are analogous. It is assumed the reader is acquainted with the general construction of general Boehmian spaces. For more details, we refer to $[1,5,7-22]$ and the references cited therein.

Let $\mathcal{G}$ be the subspace of Hartley-Hilbert transforms of members of $\mathcal{E}$. Then we say $g \in \mathcal{G}$ if there is $\varphi \in \mathcal{E}$ such that $\mathfrak{h h} \varphi=g$. Denote by $\mathfrak{H} \mathcal{D}$ the space of Hartley transforms of $\mathcal{D}$ functions. Convergence in $\mathcal{G}$ is defined as follows: $g_{i}=\mathfrak{h h} \varphi_{i} \in \mathcal{G}$, for some $\varphi_{i} \in \mathcal{E}$, is said to converge to $g \in \mathcal{G}$ if there is $\varphi \in \mathcal{E}, g=\mathfrak{h h} \varphi$ and $\varphi_{i} \rightarrow \varphi$ as $i \rightarrow \infty$.

In view of the convolution theorem, we need the following definition.

Definition 9 Let $\mathcal{D}$ be the Schwartz space of test functions of bounded support. To $\mathcal{G}$ and $\mathcal{D}$, we assign the product $x$ defined by

$$
(g \times \psi)(\xi)=\int_{0}^{\infty}(\mathfrak{p}(\varphi, \psi)(\xi) \cos (\xi t)+\mathfrak{q}(\varphi, \psi)(\xi) \sin (\xi t)) d t,
$$

where

$$
\mathfrak{p}(\varphi, \psi)(\xi)=\mathfrak{H}^{e}(\xi) \psi^{o}(\xi)+\mathfrak{H}^{o}(\xi) \psi^{e}(\xi)
$$

and

$$
\mathfrak{q}(\varphi, \psi)(\xi)=\mathfrak{H}^{e}(\xi) \psi^{e}(\xi)-\mathfrak{H}^{o}(\xi) \psi^{o}(\xi)
$$

$\mathfrak{H}^{e}\left(\mathfrak{H}^{o}\right)$ are the even and odd parts of the Hartley transform of $\varphi, g=\mathfrak{h h} \varphi$, and $\psi^{e}\left(\psi^{o}\right)$ are the even and odd parts of $\psi$, respectively. 
Thus, we have the following remark.

Remark 10 Let $g=\mathfrak{h h} \varphi \in \mathcal{E}$ and $\mathfrak{H} \psi \in \mathfrak{H} \mathcal{D}$ for some $\psi \in \mathcal{D}$, then

$$
g \times \mathfrak{H} \psi=\mathfrak{h h}(\varphi * \psi)
$$

By $\Delta$, we mean the family of all delta sequences; see [11]. Denote by $\mathfrak{H} \Delta$ the set of Hartley transforms of all members of $\Delta$. That is, if $\left(\delta_{i}\right) \in \Delta$, then $\left(\mathfrak{H} \delta_{i}\right) \in \mathfrak{H} \Delta$. Let $\left(\mathfrak{H}^{e} \delta_{i}\right)$ and $\left(\mathfrak{H}^{o} \delta_{i}\right)$ be the even and odd parts of $\left(\mathfrak{H} \delta_{i}\right)$, then it is easy to see that

$$
\left(\mathfrak{H}^{e} \delta_{i}\right)(\xi)=\int_{-\infty}^{\infty} \delta_{i}(x) \cos (x \xi) d x \rightarrow 1 \quad \text { as } i \rightarrow \infty
$$

and, similarly,

$$
\left(\mathfrak{H}^{o} \delta_{i}\right)(\xi)=\int_{-\infty}^{\infty} \delta_{i}(x) \sin (x \xi) d x \rightarrow 0 \quad \text { as } i \rightarrow \infty
$$

on compact subsets of $\mathcal{R}$.

Theorem 11 Let $g=\mathfrak{h h} \varphi$ and $\left(\delta_{i}\right) \in \Delta$, then $g \times \mathfrak{H} \delta_{i} \rightarrow g$ as $i \rightarrow \infty$.

Proof This theorem can be automatically established by invoking (25) and (26) in (24). For some detail, from (24) we have

$$
\left(g \times \mathfrak{H} \delta_{i}\right)(\xi)=\int_{0}^{\infty}\left(\mathfrak{p}\left(\varphi, \delta_{i}\right)(\xi) \cos (\xi t)+\mathfrak{q}\left(\varphi, \delta_{i}\right)(\xi) \sin (\xi t)\right) d t
$$

where

$$
\mathfrak{p}\left(\varphi, \delta_{i}\right)(\xi)=\mathfrak{H}^{e}(\xi) \mathfrak{H}^{o} \delta_{i}(\xi)+\mathfrak{H}^{o}(\xi) \mathfrak{H}^{e} \delta_{i}(\xi)
$$

and

$$
\mathfrak{q}\left(\varphi, \delta_{i}\right)(\xi)=\mathfrak{H}^{e}(\xi) \mathfrak{H}^{e} \delta_{i}(\xi)-\mathfrak{H}^{o}(\xi) \mathfrak{H}^{o} \delta_{i}(\xi)
$$

Hence, employing (25) and (26) in the above equation establishes the theorem.

Theorem 12 Let $g_{i}=\mathfrak{h h} \varphi_{i}, g=\mathfrak{h h} \varphi, g_{i} \rightarrow g$ as $i \rightarrow \infty$, and $\mathfrak{H} \psi \in \mathfrak{H} \mathcal{D}$, then

$$
g_{i} \times \mathfrak{H} \psi \rightarrow g \times \mathfrak{H} \psi \quad \text { as } i \rightarrow \infty .
$$

Proof of this theorem simply follows from (24).

Now, we state the following theorem without proof and, in fact, the proof of this theorem follows from simple computation.

Theorem 13 Let $g_{1}=\mathfrak{h h} \varphi_{1}$ and $g_{2}=\mathfrak{h h} \varphi_{2}$, then for every $\mathfrak{H} \psi \in \mathfrak{H} \mathcal{D}$ and $k \in \mathcal{R}$, we have

$$
\left(g_{1}+g_{2}\right) \times \mathfrak{H} \psi=g_{1} \times \mathfrak{H} \psi+g_{2} \times \mathfrak{H} \psi
$$


(ii)

$$
k\left(g_{1} \times \mathfrak{H} \psi\right)=k g_{1} \times \mathfrak{H} \psi=g_{1} \times k \mathfrak{H} \psi .
$$

The Boehmian space $\boldsymbol{G}_{2}(\mathcal{G}, \mathfrak{H} \mathcal{D}, \mathfrak{H} \Delta, \times)$, or $\boldsymbol{G}_{2}$, is described. Convergence in $\boldsymbol{G}_{2}(\mathcal{G}, \mathfrak{H} \mathcal{D}, \mathfrak{H} \Delta$, $\times)$ is defined as follows.

$\delta$-convergence A sequence of Boehmians $\left(\beta_{j}\right)$ in $\boldsymbol{G}_{2}(\mathcal{G}, \mathfrak{H} \mathcal{D}, \mathfrak{H} \Delta, \times)$ is said to be $\delta$ convergent to a Boehmian $\beta$ in $\boldsymbol{G}_{2}(\mathcal{G}, \mathfrak{H} \mathcal{D}, \mathfrak{H} \Delta, \times)$, denoted by $\beta_{j} \stackrel{\delta}{\rightarrow} \beta$, if there exists a delta sequence $\left(\epsilon_{i}\right)$ such that

$$
\left(\beta_{j} \times \epsilon_{i}\right),\left(\beta \times \epsilon_{i}\right) \in \mathcal{G}, \quad \forall j, i \in \mathcal{N},
$$

and $\left(\beta_{j} \times \epsilon_{i}\right) \rightarrow\left(\beta \times \epsilon_{i}\right)$ as $j \rightarrow \infty$, in $\mathcal{G}$, for every $i \in \mathcal{N}$.

$\Delta$-convergent A sequence of Boehmians $\left(\beta_{j}\right)$ in $\boldsymbol{G}_{2}(\mathcal{G}, \mathfrak{H} \mathcal{D}, \mathfrak{H} \Delta, \times)$ is said to be $\Delta$ convergent to a Boehmian $\beta$ in $\boldsymbol{G}_{2}(\mathcal{G}, \mathfrak{H} \mathcal{D}, \mathfrak{H} \Delta, \times)$ and denoted by $\beta_{j} \stackrel{\Delta}{\rightarrow} \beta$, if there exists a $\left(\epsilon_{j}\right) \in \Delta$ such that $\left(\beta_{j}-\beta\right) * \epsilon_{j} \in \mathcal{G}, \forall j \in \mathcal{N}$, and $\left(\beta_{j}-\beta\right) * \epsilon_{j} \rightarrow 0$ as $j \rightarrow \infty$ in $\mathcal{G}$.

Now, let $\boldsymbol{G}_{1}(\mathcal{E}, \mathcal{D}, \Delta, *), \boldsymbol{G}_{1}$, be the usual Boehmian space obtained from the group $\mathcal{E}$ and $\mathcal{D}$ as a subgroup of $\mathcal{E}$.

Definition 14 Let $\beta=\left[\frac{\varphi_{i}}{\delta_{i}}\right] \in \boldsymbol{G}_{1}(\mathcal{E}, \mathcal{D}, \Delta, *)$, then we define the Hartley-Hilbert transform of $\beta=\left[\frac{\varphi_{i}}{\delta_{i}}\right]$ by

$$
\mathfrak{h} \mathfrak{h}^{E} \beta=\left[\frac{\mathfrak{h} \mathfrak{h} \varphi_{i}}{\mathfrak{H} \delta_{i}}\right]
$$

in the space $\boldsymbol{G}_{2}(\mathcal{G}, \mathfrak{H} \mathcal{D}, \mathfrak{H} \Delta, \times)$.

Theorem 15 The extended Hartley-Hilbert transform $\mathfrak{h h}^{E}$ is well defined.

Proof Assume for some $\varphi_{i}, \varphi_{i}^{*} \in \mathcal{E}$ and $\delta_{i}, \delta_{i}^{*} \in \Delta, i=1,2,3, \ldots$, we have

$$
\beta:=\left[\frac{\varphi_{i}}{\delta_{i}}\right]=\left[\frac{\varphi_{i}^{*}}{\delta_{i}^{*}}\right]:=\beta^{*} \in \boldsymbol{G}_{1} .
$$

This yields $\frac{\varphi_{i}}{\delta_{i}} \sim \frac{\varphi_{i}^{*}}{\delta_{i}^{*}} ;$ i.e., $\varphi_{i} * \delta_{i}^{*}=\varphi_{i}^{*} * \delta_{i}$. An application of the Hartley-Hilbert transform on both sides and Theorem 8 together with (24) yields

$$
\mathfrak{h h} \varphi_{i} \times \mathfrak{H} \delta_{i}^{*}=\mathfrak{h} \mathfrak{h} \delta_{i}^{*} \times \mathfrak{H} \delta_{i}
$$

for $i \in \mathcal{N}$. This means $\frac{\mathfrak{h} h \varphi_{i}}{\mathfrak{H} \delta_{i}} \sim \frac{\mathfrak{h} \mathfrak{h} \varphi_{i}^{*}}{\mathfrak{H} \delta_{i}^{*}}$, i.e.,

$$
\left[\frac{\mathfrak{h h} \varphi_{i}}{\mathfrak{H} \delta_{i}}\right]=\left[\frac{\mathfrak{h} \mathfrak{h} \varphi_{i}^{*}}{\mathfrak{H} \delta_{i}^{*}}\right] .
$$


Hence,

$$
\mathfrak{h} \mathfrak{h}^{E}\left[\frac{\varphi_{i}}{\delta_{i}}\right]=\mathfrak{h} \mathfrak{h}^{E}\left[\frac{\varphi_{i}^{*}}{\delta_{i}^{*}}\right] \text {. }
$$

This proves the theorem.

Theorem 16 The operator $\mathfrak{h h}^{E}: \boldsymbol{G}_{1} \rightarrow \boldsymbol{G}_{2}$ is linear.

Proof of this theorem is obvious.

Theorem $17 \mathfrak{h h}^{E}: \boldsymbol{G}_{1} \rightarrow \boldsymbol{G}_{2}$ is one-one.

Proof Assume $\left[\frac{\mathfrak{h} h \varphi_{i}}{\mathfrak{H} \delta_{i}}\right]=\left[\frac{\mathfrak{h} \mathfrak{h} \varphi_{i}^{*}}{\mathfrak{H} \delta_{i}^{*}}\right]$ in $\boldsymbol{G}_{2}$, then certainly,

$\mathfrak{h h} \varphi_{i} \times \mathfrak{H} \delta_{i}^{*}=\mathfrak{h} \mathfrak{h} \delta_{i}^{*} \times \mathfrak{H} \delta_{i}$

By the aid of (24), we get $\mathfrak{h} \mathfrak{h}\left(\varphi_{i} * \delta_{i}^{*}\right)=\mathfrak{h} \mathfrak{h}\left(\varphi_{i}^{*} * \delta_{i}\right)$. This yields $\varphi_{i} * \delta_{i}^{*}=\varphi_{i}^{*} * \delta_{i}, \forall_{i} \in \mathcal{N}$. Thus, we have reached the identity

$$
\left[\frac{\varphi_{i}}{\delta_{i}}\right]=\left[\frac{\varphi_{i}^{*}}{\delta_{i}^{*}}\right]
$$

This completes the proof of the theorem.

Theorem 18 The mapping $\mathfrak{h} \mathfrak{h}^{E}: \boldsymbol{G}_{1} \rightarrow \boldsymbol{G}_{2}$ is continuous with respect to $\Delta$ convergence.

Proof If $\beta_{i} \stackrel{\Delta}{\rightarrow} \beta$ in $\boldsymbol{G}_{1}$ as $i \rightarrow \infty$, then we find $\left(\varphi_{i}\right) \in \mathcal{E}$ and $\left(\delta_{i}\right) \in \Delta$ such that

$$
\left(\beta_{i}-\beta\right) * \delta_{i}=\left[\frac{\varphi_{i} * \delta_{i}}{\delta_{i}}\right], \quad \varphi_{i} \rightarrow 0 \text { as } i \rightarrow \infty
$$

Upon employing $\mathfrak{h} \mathfrak{h}^{E}$ to both sides, we get

$$
\mathfrak{h} \mathfrak{h}^{E}\left(\beta_{i}-\beta\right) \times \mathfrak{H} \delta_{i}=\left[\frac{\mathfrak{h} \mathfrak{h} \varphi_{i} \times \mathfrak{H} \delta_{i}}{\mathfrak{H} \delta_{i}}\right] \equiv \mathfrak{h} \mathfrak{h} \varphi_{i} \rightarrow 0
$$

as $i \rightarrow \infty$, since $\varphi_{i} \rightarrow 0$ as $i \rightarrow \infty$. Hence, from (28) and Theorem 11, $\mathfrak{h h}^{E} \beta_{i} \rightarrow \mathfrak{h} \mathfrak{h}^{E} \beta$ as $i \rightarrow \infty$. Thus, the proof of the theorem is completed.

Theorem $19 \mathfrak{h h}^{E}$ is continuous with respect to $\delta$-convergence.

Proof If $\beta_{i} \stackrel{\delta}{\rightarrow} \beta$ as $i \rightarrow \infty$, then by [9], there are $\varphi_{j}$ and $\varphi_{i, j}$ in $\mathcal{E}$ such that $\beta_{i}=\left[\frac{\varphi_{i, j}}{\delta_{i}}\right], \beta=\left[\frac{\varphi_{j}}{\delta_{j}}\right]$, where $\varphi_{i, j} \rightarrow \varphi_{j}$ as $i \rightarrow \infty$. Continuity of $\mathfrak{h} \mathfrak{h}$ implies $\mathfrak{h h} \varphi_{i, j} \rightarrow \mathfrak{h h} \varphi_{j}$ as $i \rightarrow \infty$. Hence,

$$
\left[\frac{\mathfrak{h} h \varphi_{i, j}}{\delta_{i}}\right] \rightarrow\left[\frac{\mathfrak{h} \mathfrak{h} \varphi_{j}}{\delta_{i}}\right] \text { as } j \rightarrow \infty
$$

This theorem is completely proved. 


\section{Competing interests}

The authors declare that they have no competing interest.

\section{Authors' contributions}

Each author contributed equally in the development and preparation of the manuscript. Both authors read and approved the final copy of the manuscript.

\section{Author details}

${ }^{1}$ Current address: Department of Applied Sciences, Faculty of Engineering Technology, Al-Balqa Applied University, Amman, 11134, Jordan. ${ }^{2}$ Current address: Department of Mathematics and Institute of Mathematical Research, Universiti Putra Malaysia (UPM), 43400 UPM, Serdang, Selangor, Malaysia.

\section{Acknowledgements}

The authors express their sincere thanks to the referees for the careful and detailed reading of the manuscript and very helpful suggestions. The second author also acknowledges that the present work was partially supported by University Putra Malaysia under the grand Science Fund No: 5450536.

\section{Received: 2 October 2012 Accepted: 30 November 2012 Published: 28 December 2012}

\section{References}

1. Al-Omari, SKQ: Notes for Hartley transforms of generalized functions. Ital. J. Pure Appl. Math. 28, 21-30 (2011)

2. Sundararajan, N, Srinivas, Y: Fourier-Hilbert versus Hartley-Hilbert transforms with some geophysical applications. J. Appl. Geophys. 71, 157-161 (2010)

3. Millane, RP: Analytic properties of the Hartley transform and their applications. Proc. IEEE 82(3)، 413-428 (1994)

4. Pathak, RS: Integral Transforms of Generalized Functions and Their Applications. Gordon \& Breach, New York (1997)

5. Sundarajan, N: Fourier and Hartley transforms: a mathematical twin. Indian J. Pure Appl. Math. 8(10), 1361-1365 (1997)

6. Sundararajan, N, Srinivas, Y: Fourier-Hilbert versus Hartley-Hilbert transforms with some geophysical applications. J. Appl. Geophys. 71(4), 157-161 (2010)

7. Eltayeb, H, Kılıçman, A, Fisher, B: A new integral transform and associated distributions. Integral Transforms Spec. Funct. 21, 367-379 (2010)

8. Fisher, B, Kılıçman, A: A commutative neutrix product of ultradistributions. Integral Transforms Spec. Funct. 4(1-2), 77-82 (1996)

9. Kılıçman, A: A comparison on the commutative neutrix convolution of distributions and the exchange formula. Czechoslov. Math. J. 51(3), 463-471 (2001)

10. Kılıçman, A: On the commutative neutrix product of distributions. Indian J. Pure Appl. Math. 30(8), $753-762$ (1999)

11. Al-Omari, SKQ, Kilıçman, A: On diffraction Fresnel transforms for Boehmians. Abstr. Appl. Anal. 2011, Article ID 712746 (2012)

12. Al-Omari, SKQ, Loonker, D, Banerji, PK, Kalla, SL: Fourier sine(cosine) transform for ultradistributions and their extensions to tempered and ultraBoehmian spaces. Integral Transforms Spec. Funct. 19(6), 453-462 (2008)

13. Al-Omari, SKQ: The generalized Stieltjes and Fourier transforms of certain spaces of generalized functions. Jord. J. Math. Stat. 2(2), 55-66 (2009)

14. Al-Omari, SKQ: On the distributional Mellin transformation and its extension to Boehmian spaces. Int. J. Contemp. Math. Sci. 6(17), 801-810 (2011)

15. Al-Omari, SKQ: A Mellin transform for a space of Lebesgue integrable Boehmians. Int. J. Contemp. Math. Sci. 6(32), 1597-1606 (2011)

16. Al-Omari, SKQ: Generalized functions for double Sumudu transformation. Int. J. Algebra 6(3), 139-146 (2011)

17. Boehme, TK: The support of Mikusinski operators. Trans. Am. Math. Soc. 176, 319-334 (1973)

18. Kilıçman, A: On the Fresnel sine integral and the convolution. Int. J. Math. Math. Sci. 37, 2327-2333 (2003)

19. Kilıçman, A, Fisher, B: On the Fresnel integrals and the convolution. Int. J. Math. Math. Sci. 41, 2635-2643 (2003)

20. Mikusinski, P: Fourier transform for integrable Boehmians. Rocky Mt. J. Math. 17(3), 577-582 (1987)

21. Al-Omari, SKQ, Kılıçman, A: Note on Boehmians for class of optical Fresnel wavelet transforms. J. Funct. Spaces Appl. 2012, Article ID 405368 (2012)

22. Sundarajan, N: Tempered Boehmians and ultradistributions. Proc. Am. Math. Soc. 123(3), 813-817 (1995)

doi:10.1186/1687-1847-2012-232

Cite this article as: Al-Omari and KIlıçman: On the generalized Hartley-Hilbert and Fourier-Hilbert transforms. Advances in Difference Equations 2012 2012:232. 